\title{
Development of Efficient FPGA-Based Multi-Channel Phase Meters for IR-Interferometers
}

\author{
Luis Esteban, Student Member, IEEE, Miguel Sánchez, Member, IEEE, Juan Antonio López, Member, IEEE, \\ Petra Kornejew, Matthias Hirsch, and Octavio Nieto-Taladriz
}

\begin{abstract}
Infrared (IR) interferometry is a method for measuring the line-electron density of fusion plasmas. The significant performance achieved by FPGAs in solving digital signal processing tasks advocates the use of this type of technology in two-color IR interferometers of modern stellarators, such as the TJ-II (Madrid, Spain) and the future W7-X (Greifswald, Germany). In this work the implementation of a line-average electron density measuring system in an FPGA device is described. Several optimizations for multichannel systems are detailed and test results from the TJ-II as well as from a W7-X prototype are presented.
\end{abstract}

Index Terms-FPGA, infrared interferometry, phase meter, real-time processing.

\section{INTRODUCTION}

I

NTERFEROMETERS are devices used to measure optical path-length variations [1]. If the target of the interferometer is a plasma, these variations are proportional to the line-electron density of the plasma:

$$
\int_{0}^{L} n_{e} \mathrm{~d} l,
$$

where $n_{e}$ is the electron density and $L$ is the integration path across the plasma or chord-length. Estimating the value of $L$, the average density can be evaluated as:

$$
\left\langle n_{e}\right\rangle=\frac{\int_{0}^{L} n_{e} \mathrm{~d} l}{L} .
$$

For the reconstruction of the spatial electron density profile, $n_{e}(x, y, z, t)$ it is essential to measure several chord integrals, where the spatial reconstruction should be carried out with an inversion process over the line-integrated densities [2].
The wavelength used in the interferometer depends on several limitations. First, the maximum expected electron density must be lower than the cut-off density for that wavelength [3], which ensures that the waves can still propagate through the plasma when the maximum density is reached. Another limitation is given by refraction, which causes a deflection of the beams and eventually the loss of the interference signals. This effect is more pronounced the closer the maximum expected density is to the cut-off. However it can be moderated by reducing the wavelengths, for instance with the use of interferometers operating in the middle infrared region. With such low wavelengths mechanical vibrations are significant and a complementary interferometer is typically used to cancel these vibrations. These arrangements are called two-color interferometers (Fig. 1). Two beams are used as references and other two beams cross the plasma. When these beams reach a detector, two interference signals are generated. By comparing the phases of these signals with two sinusoidal references extracted directly from an oscillator, Fig. 1, the optical path-length variations are obtained as $\Delta \phi_{1} \lambda_{1} / 2 \pi$ and $\Delta \phi_{2} \lambda_{2} / 2 \pi$, where $\lambda_{1}$ and $\lambda_{2}$ are the wavelengths of both interferometers and $\Delta \phi_{1} / 2 \pi$ and $\Delta \phi_{2} / 2 \pi$ are the phase differences between the output signals expressed in fringes (interference and reference) of both interferometers. The final line-integrated density is evaluated as follows:

$$
\int n_{e} \mathrm{~d} l=\frac{\Delta \phi_{1} \lambda_{1}-\Delta \phi_{2} \lambda_{2}}{2 \pi p \cdot \mathrm{r}_{\mathrm{e}}\left(\lambda_{1}^{2}-\lambda_{2}^{2}\right)},
$$

where the term $\left(\Delta \phi_{1} \lambda_{1}-\Delta \phi_{2} \lambda_{2}\right) / 2 \pi$ is the compensated optical path, $p$ is the number of times that the beams cross the plasma, and $r_{e}$ is the classical electron radius.

The boxes in Fig. 1, named AOM, are acousto-optic modulators and they split the incoming beam of each interferometer into a measuring and reference beam, introducing a frequency displacement to let heterodyne detection. This is necessary because if the probing and reference beams have the same frequency, several problems arise in the interpretation of the phase shift [1], [3]. This type of arrangement is called two-color heterodyne interferometer.

In the TJ-II stellarator [4] a two-color $\mathrm{CO}_{2}-\mathrm{Nd}$ :YAG $(10.591 \mu \mathrm{m}-1.064 \mu \mathrm{m})$ single channel heterodyne interferometer is installed [5]. For several experimental campaigns an off-line phase measuring system has been used [6] and recently an FPGA-based online processing system has been tested and installed for the one-channel arrangement [7]. In addition, several tests have also been successfully carried out on the IR interferometer test-bench at IPP-Greifswald [8]. 


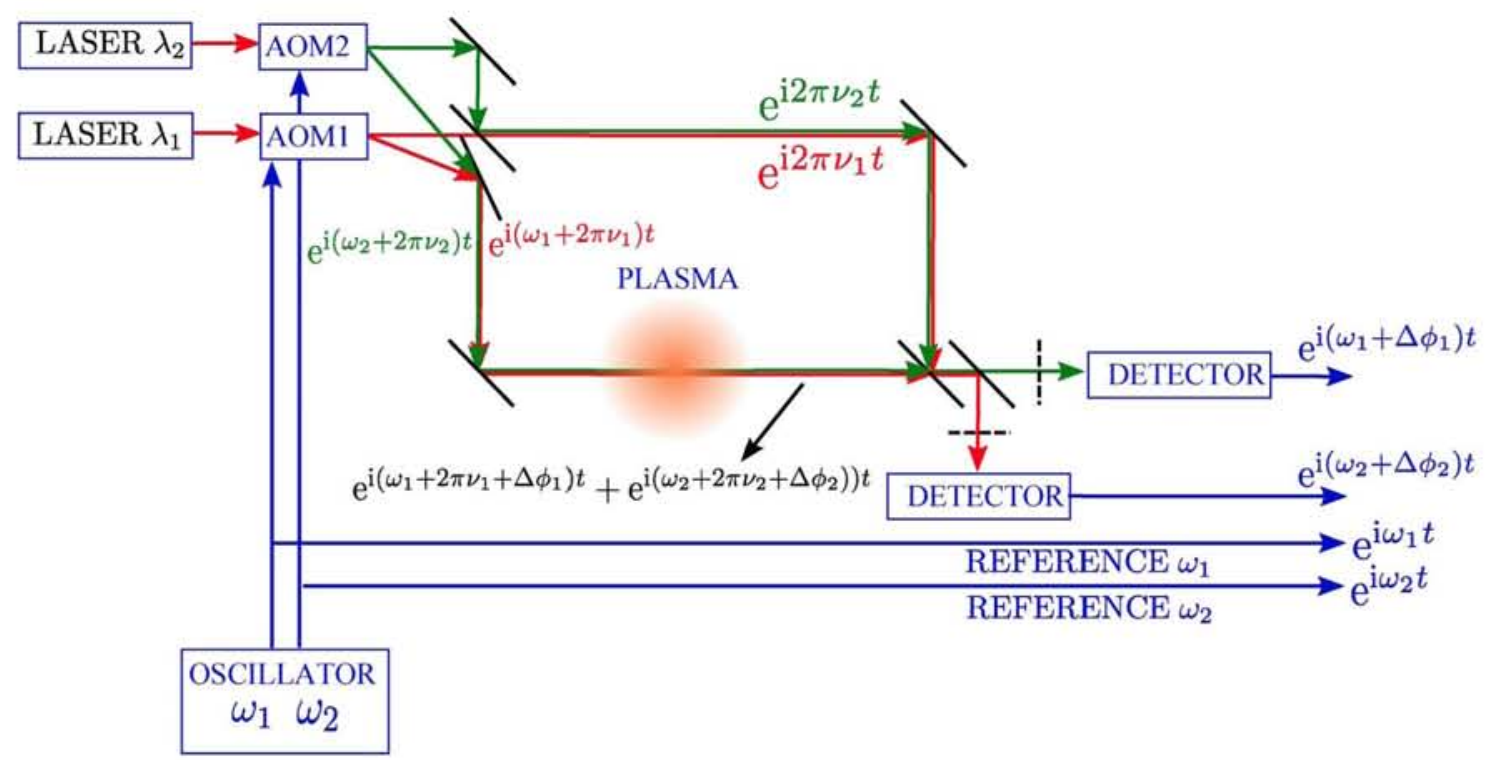

Fig. 1. Two-color heterodyne interferometer. A frequency displacement is introduced in both measuring paths using acousto-optical modulators $2 \pi \nu_{1}$ and $2 \pi \nu_{2}$. The heterodyne frequencies are $40 \mathrm{MHz}$ for all the signals, which fixes the intermediate frequencies.

Currently, an expanded beam multichannel interferometer is under development for electron density spatial profile measurements in the TJ-II stellarator [9]. In its initial version, this setup will be capable of measuring three chord integrals. For this purpose, the phase measurement must be carried out for eight signals: three $\mathrm{CO}_{2}$ interference signals, three $\mathrm{Nd}$ :YAG interference signals and two reference signals.

To perform this procedure online, very fast acquisition and processing systems are required as well as efficient signal processing algorithms. For multi-channel IR interferometers the acquisition systems must have multiple inputs, and the signals must be de-noised and processed simultaneously at very high speeds. The current digital technology allows this to be done using FPGAs, in a more flexible, accurate and faster way.

This paper is structured as follows: In Section II, a general overview of the TJ-II experiment as well as the motivation for an online average electron density measurement system are given. The general architecture of the electron density measurement system is outlined in Section III, describing several simplifications to reduce the size of the algorithms. Section IV reviews the digital processing errors that have been considered during the design of the processing system. In Section V, preliminary results obtained from the central sightline of the TJ-II expanded beam interferometer are shown, and finally in Section VI the main conclusions of this paper are summarized.

\section{General OVERVIEW OF THE TJ-II}

The TJ-II is a medium size flexible Heliac type stellarator installed in the Laboratorio Nacional de Fusion of the CIEMAT, Spain, for the study of helical axis plasmas over a wide range of parameters [4], [10]. The plasma is firstly generated using an Electron Cyclotron Resonance Heating (ECRH) heating system based on two gyrotrons working at $53.2 \mathrm{GHz}$, each one delivering a power of up to $200 \mathrm{~kW}$ to the plasma. With this method, plasmas up to average densities of $\left\langle n_{e}\right\rangle=1.7 \cdot 10^{19} \mathrm{~m}^{-3}$ can be generated [11], [12]. Beyond this value, the cut-off density for the $53.2 \mathrm{GHz}$ waves is reached and they are reflected back. To further increase the density of the plasmas, a Neutral Beam Injection (NBI) heating system ${ }^{1}$ is used. The TJ-II NBI heating system consists of two NBI injectors (hydrogen neutrals) that can deliver a power to the plasma up to $550 \mathrm{~kW}$ each [11], [12]. With this system, plasmas up to a density near to $\left\langle n_{e}\right\rangle=8 \cdot 10^{19} \mathrm{~m}^{-3}$ can be generated. Now, in order to measure in this density range the IR heterodyne interferometer described in Section I is used.

The triggering of the heating systems in the TJ-II device is pre-programmed before the plasma discharge. First starting the plasma with the ECRH heating and afterwards estimating the time when the ECRH cut-off frequency is going to be reached and triggering the NBI injectors before cut-off occurs. An online measurement of the electron density would allow to trigger the $\mathrm{NBI}$ at the optimum electron density. Furthermore this system would allow to control other diagnostics and systems of the TJ-II device.

Taking advantage of the speed of the FPGA-based processing system of the IR interferometer, an output signal is read out for control purposes in the TJ-II device. This signal, once integrated in the TJ-II control systems will allow in a first stage the triggering in real time of the NBI injectors.

\section{General ARChitecture}

The line-integrated electron density in a two-color IR interferometer is calculated according to (3), for a single line integral. As aforementioned, $\Delta \phi_{1}$ and $\Delta \phi_{2}$ are the phase differences between the output interference signals of each interferometer and their correspondent references. The calculation of these phase differences should be performed by a proper phase measuring algorithm, in this case by an interpolation algorithm [6]-[8].

\footnotetext{
${ }^{1} \mathrm{NBI}$ basically consists on launching a beam of neutral atoms into the plasma. These atoms collide with ions and electrons ionizing and increasing the plasma temperature.
} 


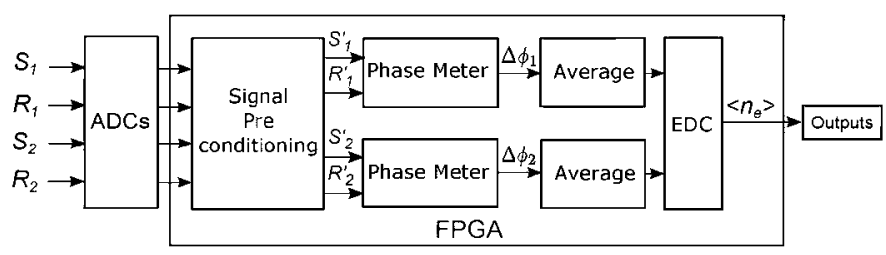

Fig. 2. Architecture of the FPGA-based processing system. $S_{1}$ and $S_{2}$ correspond to the measuring signals of $\mathrm{CO}_{2}$ and $\mathrm{NdYAG}$, respectively, of channel one, while $R_{1}$ and $R_{2}$ are the $\mathrm{CO}_{2}$ and NdYAG reference signals.

However before carrying out this process the signals must be digitized and pre-conditioned.

In the case of the TJ-II IR interferometer both the interference and reference signals have frequencies of the order of $40 \mathrm{MHz}$ (these frequencies are fixed by the AOMs). To digitize these signals, 105 MSPS, AD6645 analog-to-digital converters with a resolution of 14 bits are used. From this point the digital data is processed in a Virtex IV FPGA.

The performance of the phase measuring algorithm depends on the number of samples per period of the signals [6]-[8]. Considering directly the signals at the output of the ADCs, the value of this parameter is $105 / 40=2.625$ Samples/period, which is below the minimum number required, $(>4)$ for the interpolation algorithm to work correctly [6]. Then, it is necessary to increase the number of samples per period of the signals. Furthermore, the input signals are likely to be noisy, thereby necessitating some digital filtering before computing the phase differences. Fig. 2 illustrates the complete average electron density calculation, in which the above tasks are performed in the signal pre-conditioning block. After calculating the phase differences, an averaging procedure is performed to reduce the random noise that is typically present in $\Delta \phi_{1}$ and $\Delta \phi_{2}$. Finally the electron density is computed (electron density computation block, EDC) and the data is send to memory and to the TJ-II systems.

In the remaining parts of this Section, the architecture of the electron density processing system is described. The pre-conditioning stage is explained in Section III-A, while the phase measuring algorithm is reviewed in Section III-B. In this Subsection, several simplifications are considered, and the averaging procedures and the final average density computation are described. In Section III-C, the generation of the digital control signal is considered, and finally in Section III-D the integration of the measurement system with the multichannel interferometer is outlined.

\section{A. Pre-Conditioning Stage}

In this stage two operations on the signals are carried out. First, the input digitized signals are filtered and then a sampling rate alteration procedure is performed to increase the number of samples per period. For the first one, the phase response of the filters should be linear and with the same group delay for all signals [8]. In this sense, Finite Impulse Response (FIR) bandpass filters with linear phase response have been used.
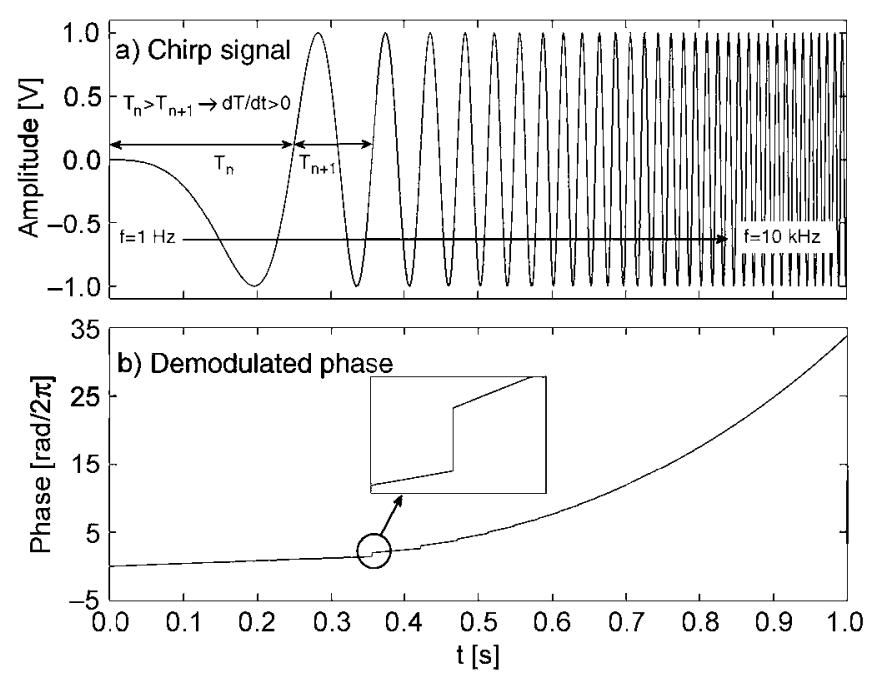

Fig. 3. Chirp signal with quadratic frequency sweep starting at $1 \mathrm{~Hz}$ at time $t=0 \mathrm{~s}$, and finishing with a frequency of $100 \mathrm{~Hz}$ at time $t=1 \mathrm{~s}$. The sampling frequency is $10 \mathrm{kHz}$. It can be observed that when $T_{n}$ differs significantly from $T_{n+1}$ an error is introduced in the demodulated phase. This error is eliminated using the current period for the computation of the division in (5).

After filtering, the number of samples per period of the signals must be increased. Since the output signals of the interferometer are bandpass signals, the number of samples per period can be increased easily by the alteration of the sampling rate period of the processing system. This is performed by first under-sampling the original signals and then up-sampling (zero padding) and filtering the signals to recover the original sampling rate. The basic sampling rate alteration blocks, down-sampler and up-sampler are well documented in the literature [13]-[15]. If $N$ is the under-sampling factor and $L$ the up-sampling factor, the final number of samples per period is given by:

$$
M=\frac{F_{s} / N}{f_{c}-F_{s} / N} \cdot L,
$$

where $F_{s}$ is the sampling frequency and $f_{c}$ is the carrier frequency of the signals. In the case of the TJ-II interferometer, $F_{s}=105 \mathrm{MSPS}$ and $f_{c}=40 \mathrm{MHz}$, taking $N=L=3$ results in a total number of samples per period of 21 , which is a suitable number for the phase measuring algorithm to work. This procedure digitally down-shifts the frequency of the inputs signals to an equivalent frequency of $5 \mathrm{MHz}$.

It is worth mentioning that the design of this stage depends on the detection configuration. In general, two detectors are used to detect the interference signals as shown in Fig. 1. However, in the particular case of the IPP-Greifswald IR interferometer test-bench, a single detector can be used to detect both interference signals (40 MHz and $25 \mathrm{MHz}$ ) [16]. In this case both signals must be separated, what can be done digitally using two FIR filters, that in this case would have a double functionality, i.e split both frequency carriers (diplexion) [8], and reduce the noise of the signals as explained above. 


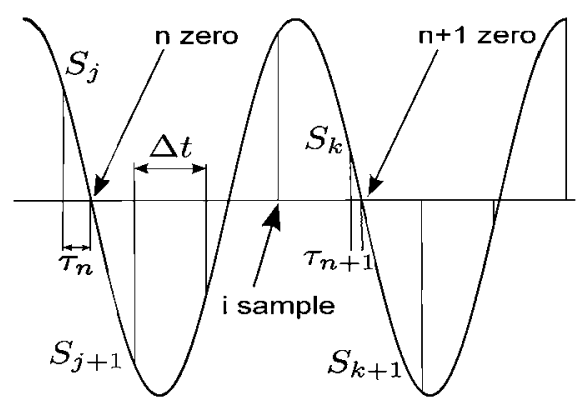

Fig. 4. Parameters used in the phase measuring interpolation algorithm.

\section{B. Phase Measuring Algorithm}

In [6], [7], [17] the implementation of a novel phase measuring interpolation algorithm was described. According to this algorithm, the phase of a digitized signal can be computed as:

$$
\varphi_{i}=2 \pi n+\frac{2 \pi\left[(i-j) \Delta t-\tau_{n+1}\right]}{t_{n}-t_{n+1}}
$$

where the factor $n$ is the number of zero crossings. The term $\tau_{n}$ corresponds to a linear interpolation around the $n^{\text {th }}$ zero, as shown in Fig. 4. It is calculated according to the absolute values of the former and latter samples of a zero cross as:

$$
\tau_{n}=\frac{\left|S_{j}\right|}{\left|S_{j}\right|+\left|S_{j+1}\right|} \Delta t
$$

where $j$ is the number of samples acquired until the $n^{\text {th }}$ zero cross and $i$ is the current sample number. The terms $t_{n}$ and $t_{n+1}$ are the total times at the current zero and at the next zero cross:

$$
\begin{aligned}
t_{n+1} & =j \Delta t+\tau_{n+1}, \\
t_{n} & =k \Delta t+\tau_{n},
\end{aligned}
$$

where $k$ is the number of samples that will be acquired at the next zero cross. Since this algorithm is non-causal, in [7] it uses the values of the former period to compute the current period parameters provided that $t_{n} \approx t_{n+1}$. This approach introduces an error that increases with increasing phase velocity $\mathrm{d} \phi / \mathrm{d} t$. This effect is illustrated in Fig. 3. Another approach is to introduce a latency higher than the signals period in the processing system, to solve the causality problem. This is being implemented currently for the expanded beam phase detection system.

A very important issue that arises in upgrading a processing system for a single channel to a multichannel interferometer scheme is that the implementation size begins to approach the maximum capacity of the FPGA. In general, the complete version of the interpolation algorithm requires the computation of two divisions as shown in Fig. 5. A simplification, which consists of suppressing the zero crossing interpolation and interpolating the intermediate signals between zero crossings as:

$$
\varphi_{i}=2 \pi n+\frac{2 \pi(i-j) \Delta t}{t_{n}-t_{n+1}}
$$

can be made, where:

$$
\begin{aligned}
t_{n+1} & =j \Delta t, \\
t_{n} & =k \Delta t .
\end{aligned}
$$

With this simplification the signal-to-noise ratio of the output phase difference signals is made slightly worse. In practice, the phase noise is of a random walk nature, so by filtering or averaging the correspondent phase difference signals these noises are suppressed [7]. By having an even faster system the phase differences can be computed by just counting the zero crossings of the signals. The resolution achieved depends on the averaging factor as $1 / \sqrt{N}$. This is the simplest algorithm realizable and its main drawback is that the sampling frequencies of the ADCs must be higher than those of the other algorithms explained in order to obtain the same final resolution. The main advantage of this approach is that the size of the algorithm is minimal so other hardware in the FPGA may be used for other tasks.

\section{Digital Control Signal}

After computing the phase differences, the calculation of the average electron density is straight-forward. At this point this signal is stored in an FPGA on-board memory, and later transferred to the TJ-II database. Also, a signal is read out of the FPGA during the plasma discharge to be used by the TJ-II control systems and other diagnostics. For the latter, a general purpose input/output 14-bit digital port, (GPIO) is used. This port interfaces directly with the FPGA I/O pins. The data is sent out directly, in parallel, through this port, together with a synchronization clock, every $1 \mathrm{~ms}$, which is equal to the responses time of the TJ-II heating and control systems.

\section{Global Design}

Two detectors arrays are used for the $\mathrm{CO}_{2}$ and NdYAG beams respectively. The electron density (3) is calculated for three chord integrals in the FPG $\Lambda$, so the final design consists of a repeated sequence of the blocks needed for the phase measurement of one channel. In Figs. 5 and 6 the processing pipelines corresponding to the complete version of the phase measuring algorithm and the simplified one are shown. As shown in Table I, the implementation of the complete algorithm requires significantly more hardware resources. For instance, for three chord integrals, 19840 FPGA slices are used out of 44416 for the complete algorithm. Fig. 7 shows the complete processing system hardware. It includes the detection arrays, the pre-amplification stage, and the integration of the whole system in the TJ-II global acquisition system.

\section{ERROR SOURCES}

The use of infrared interferometry for the line-electron density measurement requires of the implementation of precise phase meters. For instance, in the case of the TJ-II IR interferometer, a plasma with a density of $3 \cdot 10^{18} \mathrm{~m}^{-3}$ requires a precision in the measurement of the optical path-length differences $\left(\lambda_{1} \Delta \phi_{1} / \pi\right.$ and $\left.\lambda_{2} \Delta \phi_{2} / \pi\right)$ of $0.02 \mu \mathrm{m}, 1 / 500$ of a $\mathrm{CO}_{2}$ fringe. It is essential to minimize systematic error sources, and to evaluate globally the errors made in the measurements. 


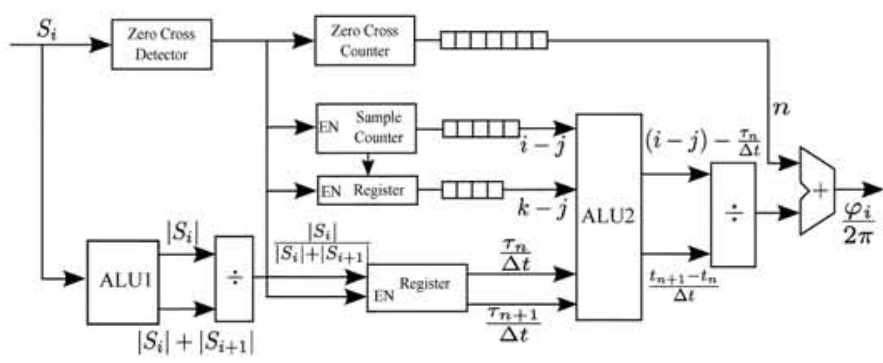

Fig. 5. Block diagram of the FPGA implementation of the complete phase measurement algorithm. The squares following the Zero Cross Counter are used to equalize the pipeline.

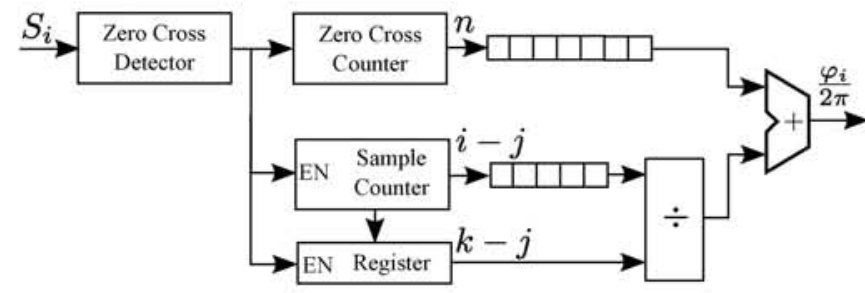

Fig. 6. Block diagram of the FPGA implementation of the simplified phase measurement algorithm. Note: That in this case only, one divider is needed.

TABLE 1

HARDWARE RESOURCES OF A VIRTEX IV FPGA USED IN THE IMPLEMENTATION OF THE LINE-INTEGRATED DENSITY MEASUREMENT SYSTEM, FOR ONE OPTICAL. CHANNEL.

\begin{tabular}{ccc}
\hline Hardware resource & Complete algorithm & Simplified algorithm \\
\hline \hline Slices & 5552 & 2480 \\
FFs & 7730 & 3708 \\
LUTs & 7415 & 3304 \\
EMB MULTs & 10 & 10 \\
\hline
\end{tabular}

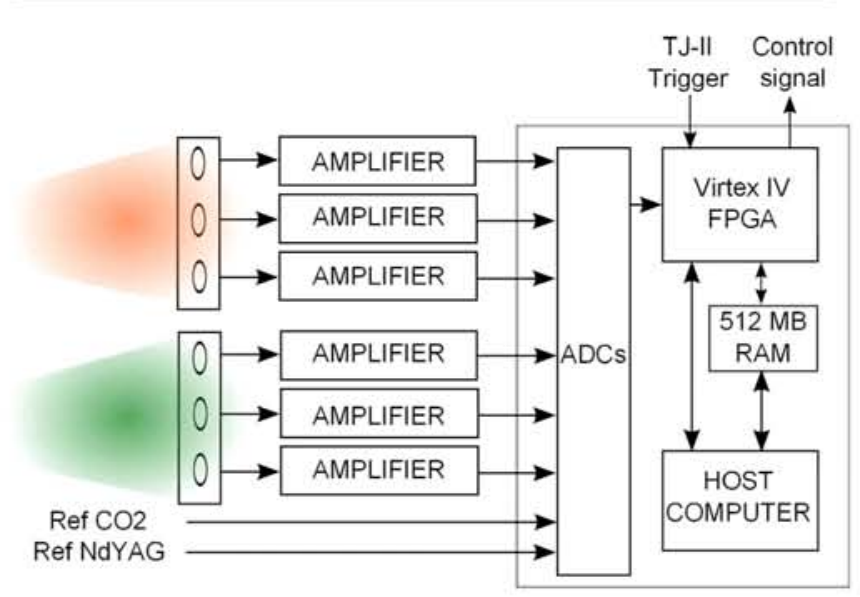

Fig. 7. FPGA-based processing system hardware. The processing parameters are controlled by a Host Computer through a compact PCI bus. This computer also has access to the data stored in the onboard RAM memory by the FPGA (line-integrated density signals).

Interferometers are affected by errors and noises that are in general of different nature, and in some cases are related. Here the electrical crosstalk, the fringe jump problem and the finite word-length effects are briefly reviewed.

The electrical crosstalk is the undesired coupling between the output signals of the interferometer [18]. If these signals have different frequencies then the crosstalk can be removed,

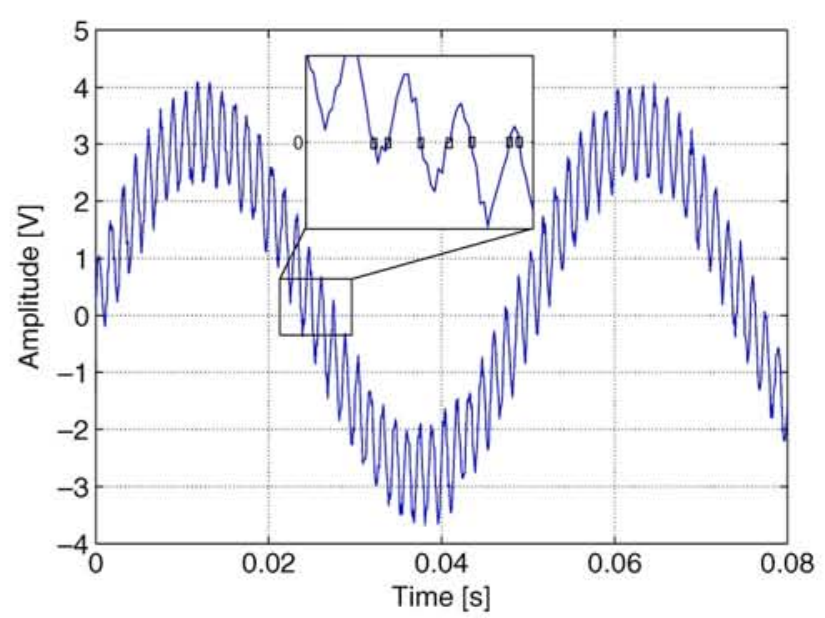

Fig. 8. Example of a noisy signal in which the exact position of the zero crossings cannot be distinguished.

using for instance bandpass filters. It should be taken into account that electrical crosstalk usually originates in the analog pre-processing electronics, so it is desirable to reduce it as much as possible. The crosstalk should be taken into account specially in multi-channel system in which each signal may be a weighted mixture of the rest of the output signals of the interferometer. In [18] a crosstalk reduction algorithm is described for the one-channel case.

As previously mentioned the phase measurement performed in fusion interferometers requires tracking of the zero crossings of the signals, (9). One of the errors mentioned above is related to the presence of fringe jumps in the phase measurements, which are due to erroneous counting of zero crossings that result in a cumulative error multiple of $2 \pi$.

This phenomena can be important when counting the zero crossings of noisy signals, as illustrated in Fig. 8. In this case the phase measurement procedure would lead to the counting of seven zero crossings instead of one, as can be seen in the detail of the figure, thereby introducing an error of $12 \pi$ in the measurement. This type of noisy signal can be avoided by filtering, as is done in the case of the TJ-II device in the pre-conditioning stage, Section III-A. The presence of fringe jumps can also originate as plasma events or as misalignments of the interferometers [8], [19], which has conducted to the development of fringe jump correction systems in several fusion interferometers [20]-[22]. It is also worth mentioning that the phase measuring algorithm described in Section III-B has been used for several campaigns at the TJ-II IR interferometer and no fringe jumps have been observed. The line-electron density measurement must be free of fringe jumps in order to provide a real time control signal.

The implementation of DSP algorithms in FPGAs usually requires the translation of a floating-point calculation into a fixed-point one. This causes the apparition of finite word-length effects, that degrade the performance of the application. The most important one is the round-off-noise (RON) [23], which causes the apparition of an additive white noise. This is caused due to the truncation or rounding of the signals least significant bits [23]. A trade off between word-length and output signal-to-noise ratio must be established. In this sense, a study of 


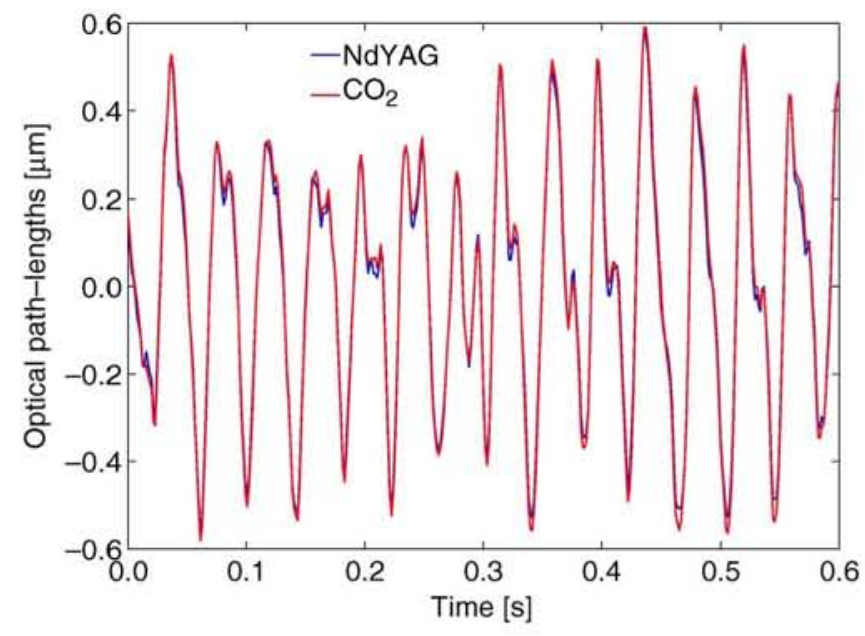

Fig. 9. Path-length differences for the NdYAG beam (blue line) and $\mathrm{CO}_{2}$ (red line). It can be seen that both traces have an oscillation of approximately $0.7 \mu \mathrm{m}$ (pk-pk). These oscillations are present in the optical table and should be compensated with a precision of at least 1/400 of fringe in the phase measurements for the TJ-II typical ECRH plasma densities.

the propagation of the RON through the non-linear operations shown in Figs. 5 and 6 is essential [24] to decide the optimal word-lengths.

The errors and noises summarized above have been considered during the development of the measuring system, currently a crosstalk correction system for multichannel systems is being developed. In the next Section it is shown that the errors are under acceptable bounds. It should be considered that these sources of error are not the only ones that affect the interferometer performance. Furthermore, the optical setup is affected by several sources of error and noises, but this study is beyond the scope of this paper. FPGA-based processing systems have the advantage that some of these errors can be corrected in real time.

\section{RESULTS}

The complete expanded-beam, multi-channel heterodyne, IR interferometer system has been installed recently in the TJ-II experimental hall. This setup includes the complete optical setup as well as the ultra-fast FPGA-based phase measuring system for the average electron density calculation. This system has been implemented following the methodology described above, with the final design having a total latency of $80 \mu \mathrm{s}$, that is fixed by the averaging factor, in this case 8192 . The output bandwidth of the electron density signals is $6.5 \mathrm{kHz}$ and the control signal is read out every $1 \mathrm{~ms}$. The whole system is controlled during plasma operation through a cPCI bus. A similar system has been also tested in the IPP-Greifswald interferometer prototype, with the particularity that the input filters are also used to split two frequency carriers (diplexion), as explained in Section III-A. The latency and output bandwidth are similar as the ones obtained with the TJ-II IR interferometer measuring system.

In Fig. 10, the first results without plasma obtained by the expanded beam interferometer are presented. The data corresponds to the central expanded beam sightline. By subtracting both traces the compensated path-length is obtained (Fig. 11(a)). Under plasma conditions this compensated optical-path would

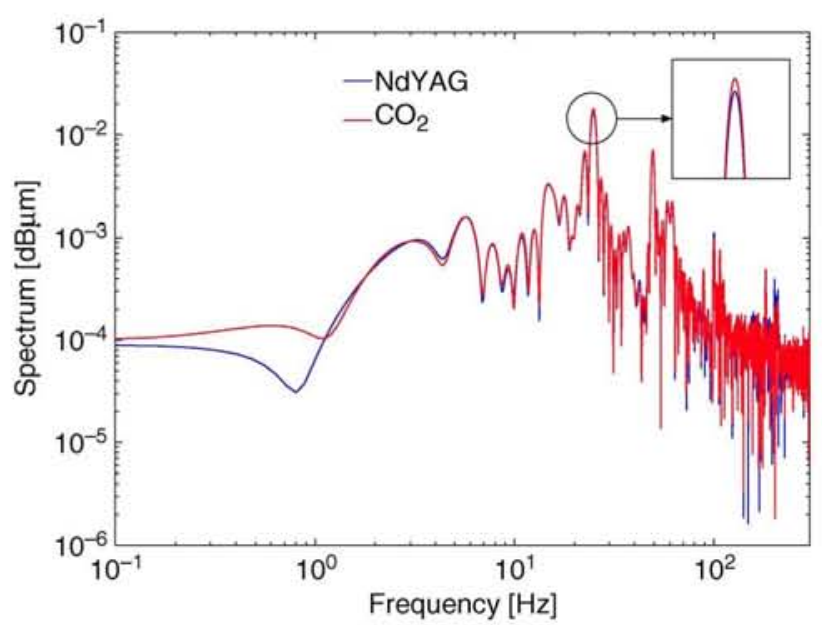

Fig. 10. Spectrum of the optical path-length of the NdYAG and $\mathrm{CO}_{2}$ signals of Fig. 9. In the detail of the figure, it is shown that the optical paths are not completely compensated. The high frequency noise is of random walk type in both signals.

be proportional to the line-integrated plasma density (3), provided that the noise is within acceptable bounds. In Fig. 10, the spectrums of the optical path signals of NdYAG and $\mathrm{CO}_{2}$ are illustrated. As can be seen in this Figure, the high frequency noise is of random walk type, which justifies the averaging procedure carried out before the subtraction of the optical paths to reduce the noise. Also it can be seen how the traces are not completely equal. The subtraction is shown in Fig. 11(a), where it can be seen how this discrepancy in the phase differences affects the compensated path with the apparition of a $25 \mathrm{~Hz}$ deterministic signal. This signal has been removed with a notch filter of $5 \mathrm{~Hz}$ bandwidth. However, this is not ideal, rather this peak should be removed by achieving a better alignment of the interferometer or by applying more refined signal separation DSP techniques. Similar results have been obtained in the IPP Greifswald interferometer test-bench [8].

In Fig. 12 plasma density of TJ-II discharge number 18040 is shown, where the measurements correspond to the $\mu$ wave interferometer, red trace and IR-interferometer, blue trace. As seen in the Fig. 12 the traces are different. This is because there exist uncorrected fringe jumps in the $\mu$ wave interferometer. The $\mu$ wave interferometer uses an IQ phase detection system with no fringe jump correction system so they are post-corrected. In the case of the interpolation algorithm used for the IR-interferometer no fringe jumps have been observed because of a failure in the phase measuring system. For control purposes it is essential that the electron density signal is free of such fringe jumps.

\section{CONCLUSIONS}

An online processing system based on a Virtex IV FPGA and an interpolation algorithm have been tested in the TJ-II singlechannel IR-Interferometer, in the expanded beam interferometer, and in the W7-X IR-interferometer benchmark with good results in each case. A new version of the algorithm has been proposed to reduce its size while achieving acceptable accuracy. The preliminary multi-channel design, including three channels, now fits in the FPGA. There are also additional resources to implement other DSP algorithms to reduce the sources of error 


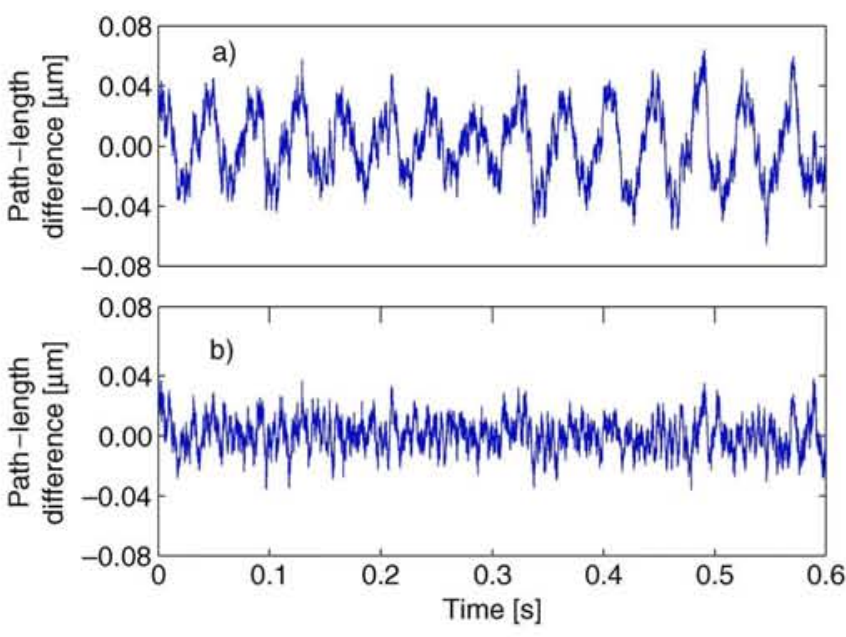

Fig. 11. a) Compensated raw optical-path correspondent to the substraction $\lambda_{\mathrm{CO}_{2}} \Delta_{\mathrm{CO}_{2}}-\lambda_{\mathrm{NdYAG}} \Delta_{\mathrm{NdYAG}} ;$ an spurious deterministic signal is coupled into the final compensated signal. In b) this spurious signal has been removed using a $5 \mathrm{~Hz}$ bandwidth notch filter.

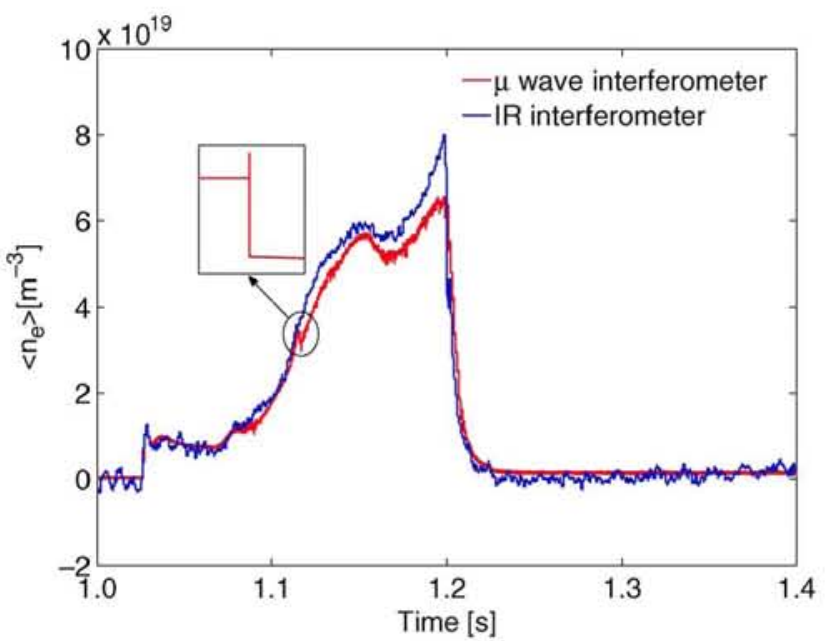

Fig. 12. Data correspondent to the line-integrated electronic density measurement performed by the TJ-II one channel IR-interferometer (blue line) and by the $\mu$ wave interferometer (red line). The data corresponds to the shot number 18040 .

explained in Section IV, particularly the crosstalk. The preprocessing stage, in which a pre-filtering and a frequency digital down-conversion to the input signals is applied, has increased the performance of the phase measuring algorithm. A crosstalk removal procedure based on the ideas depicted in Section IV is also going to be implemented in this pre-processing stage.

With the online processing system based on FPGAs, joined to a multi-channel interferometer, it is possible to process in real time the final line-integrated electron density signals, and also to perform a reconstruction of the plasma spatial profile.

Thanks to the FPGA-based online processing system, it is possible to process multiple signals in parallel, what allows to compute the spatial electron density profile in multi-channel interferometers in real time.

For these purposes, it would be desirable to increase the capabilities of the system, especially the analog-to-digital converter resolution and velocity, to increase the FPGA resources and the SDRAM memory capacity, which are the main bottlenecks of our current system. In this sense, it will be upgraded to a system based on the Advanced Telecommunication Computing Architecture (ATCA), which has the capability of sampling up to 400 MSPS with a resolution of 14 bits, and two on-board Virtex IV FPGAs as well as $2 \mathrm{~GB}$ of onboard SDRAM memory [25], [26]. These boards will significantly increase the processing system capabilities.

\section{ACKNOWLEDGMENT}

The author would like to thank K. J. McCarthy and the anonymous reviewers for their comments and suggestions, and also D. Alegre, A. Bustos, T. Happel, and the TJ-II, UPM-LSI and W7-X teams for their support.

\section{REFERENCES}

[1] I. H. Hutchinson, Principles of Plasma Diagnostics. Cambridge, U.K.: Cambridge Univ. Press, 1990.

[2] T. Kirimoto, K. Danial, T. Amishima, and A. Okamura, "Performance analysis of independent component analysis to separate mixtures of complex sinusoidal signals," in Proc. SICE Annu. Conf., Tokyo, Japan, 2008.

[3] H. J. Hartfuss, T. Geist, and M. Hirsch, "Heterodyne methods in millimetre wave plasma diagnostics with applications to ece, interferometry and reflectometry," Plasma Phys. Control. Fusion, vol. 39, no. 11, p. $1693,1997$.

[4] C. Alejaldre, "Tj-ii project: A flexible heliac stellarator," Fusion Technol., vol. 17, 1989.

[5] P. Acedo, H. Lamela, M. Sanchez, T. Estrada, and J. Sanchez, " $\mathrm{CO}_{2}$ $\left(\lambda_{m}=10.6 \mu \mathrm{m}\right) \mathrm{He}-\mathrm{Ne}\left(\lambda_{m}=10.6 \mathrm{~nm}\right)$ two-color laser interferometry for low and medium electron density measurements in the TJ-II stellarator," Rev. Sci. Instrum., vol. 75, no. 11, pp. 4671-4677, 2004.

[6] M. Sanchez, J. Sanchez, T. Estrada, E. Sanchez, P. Acedo, and H. Lamela, "High resolution co[sub 2] interferometry on the TJ-II stellarator by using an ADC-based phase meter," Rev. Sci. Instrum., vol. 75 , no. 10 , pp. 3414-3416, 2004.

[7] L. Esteban, M. Sánchez, J. A. López, O. Nieto-Taladriz, and J. Sánchez, "Continuous plasma density measurement in TJ-II infrared interferometer-advanced signal processing based on FPGAs," Fusion Eng. Des., submitted for publication.

[8] L. Esteban, M. Sánchez, J. Sánchez, P. Kornejew, M. Hirsch, J. A. López, A. Fernández, and O. Nieto-Taladriz, "Continous phase measurement in the W7-X IR-interferometer by means of an FPGA and high speed ADCS," Fusion Sci. Technol., submitted for publication.

[9] P. Pedreira, L. Esteban, A. R. Criado, P. Acedo, M. Sánchez, and J. Sánchez, "Two color multichannel heterodyne interferometer set up for high spatial resolution electron density profile measurements in TJ-II," Rev. Sci. Instrum., vol. 81, no. 10, p. 10D517, 2010.

[10] C. Alejaldre, "TJ-II the flexible heliac," Europhys. News, pp. 232-233, 2005.

[11] J. Guasp, S. Petrov, J. M. Fontdecaba, and V. Tribaldos, "Ion confinement studies in NBI heated TJ-II plasmas using CX-NPA diagnostics," in Proc. 36th EPS Conf. Plasma Physics, Sofia, Bulgaria, 2008, vol. 33E, p. 4179.

[12] M. Liniers, M. Ochando, F. L. Tabarés, D. Tafalla, J. Guasp, R. Jiménez-Gómez, F. Castejón, D. López-Bruna, A. López-Fraguas, I. Pastor, A. Cappa, C. Fuentes, J. M. Fontdecaba, E. Ascasíbar, and T. Estrada, and the TJ-II Tea, "Global confinement in NBI plasmas of the TJ-II stellarator under lithium-coated wall conditions," in Proc. 36th EPS Conf. Plasma Physics, Sofia, Bulgaria, 2009, vol. 33E, p. 4174.

[13] S. K. Mitra, Digital Signal Processing. New York: McGraw-Hill, 2006.

[14] B. Farhang-Boroujeny, Signal Processing Techniques for Software Radios. Raleigh, NC: Lulu Publishing, 2010.

[15] J. G. Proakis and D. K. Manolakis, Digital Signal Processing. Upper Saddle River, NJ: Prentice Hall, 2006.

[16] P. Kornejew, M. Hirsch, T. Bindemann, A. Dinklage, H. Dreier, and H. J. Hartfuss, "Design of multichannel laser interferometry for W7-X," Rev. Sci. Instrum., vol. 77, p. 10F128, 2006.

[17] P. V. Zubarev and A. D. Khilchenko, "Precision phase discriminator for the heterodyne interferometric plasma-density measurement technique," Instrum. Exp. Tech., vol. 46, pp. 171-176, 2003, 10.1023/A:1023661531511. 
[18] M. Sanchez, L. Esteban, P. Kornejew, and M. Hirsch, "Admissible crosstalk limits in a two colour interferometer for plasma density diagnostics. A reduction algorithm," in Proc. AIP Conf., 2008, vol. 993, no. 1 , pp. $187-190$.

[19] A. Murari, L. Zabeo, A. Boboc, D. Mazon, and M. Riva, "Real-time recovery of the electron density from interferometric measurements affected by fringe jumps," Rev. Sci. Instrum., vol. 77, no. 7, p. 073505, 2006.

[20] C. Gil, A. Barbuti, D. Elbèze, P. Pastor, J. Philip, and L. Toulouse, "Fringe jump analysis and electronic corrections for the tore supra far infrared interferometer," Rev. Sci. Instrum., vol. 79, no. 10, p. 10E710, 2008.

[21] J.-W. Juhn, K. C. Lee, Y. S. Hwang, C. W. Domier, Jr., N. C. Luhmann, B. P. Leblanc, D. Mueller, D. A. Gates, and R. Kaita, "Fringe-jump corrected far infrared tangential interferometer/polarimeter for a real-time density feedback control system of NSTX plasmas," Rev. Sci. Instrum., vol. 81, no. 10, p. 10D540, 2010.

[22] C. Gil, A. Barbuti, P. Spuig, A. Boboc, and S. Dorling, JET EFDA Contributors, "Analysis and improvements of fringe jump corrections by electronics on the jet tokamak far infrared interferometer," Rev. Sci. Instrum., vol. 81, no. 10, p. 10D536, 2010.
[23] J. A. Lopez, C. Carreras, and O. Nieto-Taladriz, "Improved intervalbased characterization of fixed-point 1ti systems with feedback loops," IEEE Trans. Comput.-Aided Design Integr. Circuits Syst., vol. 26, no. 11, pp. 1923-1933, Nov. 2007.

[24] L. Esteban, J. A. López, A. Fernández, C. Carreras, G. Caffarena, C. A. López, O. Nieto-Taladriz, and M. Sánchez, "Round-off noise estimation of fixed-point algorithms using polynomial chaos expansion," IEEE Trans. Circuits Syst. I, Reg. Papers, page Under prepraration.

[25] B. B. Carvalho, A. J. N. Batista, M. Correia, A. Neto, H. Fernandes, B. Gonçalves, and J. Sousa, "Reconfigurable atca hardware for plasma control and data acquisition," Fusion Eng. Des., vol. 85, no. 3-4, pp. 298-302, 2010.

[26] R. C. Pereira, J. Sousa, A. M. Fernandes, F. Patrício, B. Carvalho, A. Neto, C. A. F. Varandas, G. Gorini, M. Tardocchi, D. Gin, and A. Shevelev, "ATCA data acquisition system for gamma-ray spectrometry," Fusion Eng. Des., vol. 83, no. 2-3, pp. 341-345, 2008. 